\title{
An approximate method for evaluating the shear band thickness in saturated soils
}

\author{
X. B. Lu ${ }^{1, * \dagger}$, Wang Shuyun ${ }^{1}$, Wang Yihua ${ }^{1}$ and Peng $\mathrm{Cui}^{2}$ \\ ${ }^{1}$ Institute of Mechanics, Chinese Academy of Sciences, 100080 Beijing, China \\ ${ }^{2}$ Institute of Mountain Hazard and Environment, Chinese Academy of Sciences, 610041 Chengdu, China
}

\begin{abstract}
SUMMARY
A formula for the thickness of a shear band formed in saturated soils under a simple shear or a combined stress state has been proposed. It is shown that the shear band thickness is dependent on the pore pressure properties of the material and the dilatancy rate, but is independent of the details of the combined stress state. This is in accordance with some separate experimental observations. Copyright (C) 2004 John Wiley \& Sons, Ltd.
\end{abstract}

KEY WORDS: shear band thickness; saturated soils; combined stresses; simple shear

\section{INTRODUCTION}

Shear bands in saturated soils are narrow zones which have been observed during simple shear or plane strain or triaxial compressive experiments (e.g. References [1-5]). From; these experiments closed formulas and diagrams for the inclination angle of critical discontinuity surfaces were derived. These experimental results characterize the behaviour of a loose, finegrained, water-saturated sand tested under globally undrained conditions. For the local measurements of boundary forces and deformations, stereophotogrammetry, digitized optical images [6], X-ray method and optical measurements [7,8] are used to track the progressive localization of strain and the influences of factors. It was found that the normalized shear band thickness decreases as grain-size increases and as density decreases and is dependent on the specimen dilatancy angle. Lade and Qiong Wang [9] performed true triaxial tests on tall prismatic specimens of dense Santa Monica Beach sand in a modified version of a cubical triaxial apparatus to study the effect of shear banding on failure in the full range of the

\footnotetext{
*Correspondence to: X. B. Lu, Institute of Mechanics, Chinese Academy of Sciences, 100080 Beijing, China.

†E-mail: xblu@imech.ac.cn

Contract/grant sponsor: National Natural Science Foundation-Key Youth Foundation; contract/grant number: 40025103

Contract/grant sponsor: National Natural Science Foundation; contract/grant number: 10202024
}

Copyright (C) 2004 John Wiley \& Sons, Ltd.

Received 17 May 2004

Revised 16 August 2004 
intermediate principal stress. Mooney et al. [10] investigated the development of localized strains in soils. Borja and Lai [11] investigates the impact of strain localization in the form of strong discontinuity, or displacement jump, on the limit strengths of retaining walls supporting an elastoplastic backfill.

The theoretical investigations (e.g. References [12-15]) have been processed to analyse the inception of localization as a bifurcation or non-uniqueness of the solution for homogeneous deformation. In these analyses, the saturated soils [16-21] are taken as a two-phase continuum and to investigate the instability under undrained deformation. Their investigations focus on two major bifurcation phenomena i.e. shear-banding and liquefaction. The shear band thickness was analysed based on the Cosserat constitutive relation [22]. de Borst [23] viewed the kinematics of a shear band as a discontinuity in a solid. The so-called strong and weak discontinuity models are discussed and their interrelation is shown. From the analysis reported by $\mathrm{Lu}$ [24], the evolution of the shear band was only discussed in a simple condition.

Shear localization along these bands is caused by the destabilizing influence of the pore pressure increase (or effective stress decrease) on the plastic flow. Under normal circumstances, saturated soils harden as the strain and strain rate increase and the yield stress decreases with increasing pore pressure. Thus, it is possible for the pore pressure softening effect to more than compensate for the hardening effects of the strain and strain rate causing the strain to localize eventually in one or more bands of intense shear.

The plastic work (which causes the pore pressure increase and soil dilatancy) and the pore pressure diffusion properties are observed to be of fundamental importance to the appearance and the geometry of shear bands. Indeed, the plastic work leads to shear localization, while pore pressure diffusion leads to shear bands extending [25]. The bands generally result from loadings that cause high local strains. Shear bands in saturated soils are usually very narrow, of the order of less than 20 radii of the soil particles (e.g. $[6,13])$.

Because of the complexity of the problem, a lot of researchers are investigating it by using numerical modelling methods [26-28]. They investigate the incipience of shear band with an incrementally non-linear constitutive equation. Necessary conditions for the emergence of shear band are derived. In order to overcome the difficulty of grid-dependence when softening takes place, many numerical methods and constitutive relations are presented [29]. Nevertheless, there is no convenient and exact method to estimate the shear band thickness in saturated soils.

On the above viewpoint, an approximate approach is presented to estimate the shear band thickness in simple shear and multidimensional stress states in this paper.

\section{FORMULATIONS}

In this paper, our aim is to find a convenient way to evaluate the band thickness in saturated soils. We first consider a simple shear (Figure 1(a)) to discuss the one-dimensional shear band thickness, and then consider a plane strain problem (Figure 1(b)) to discuss the multidimensional band thickness. 

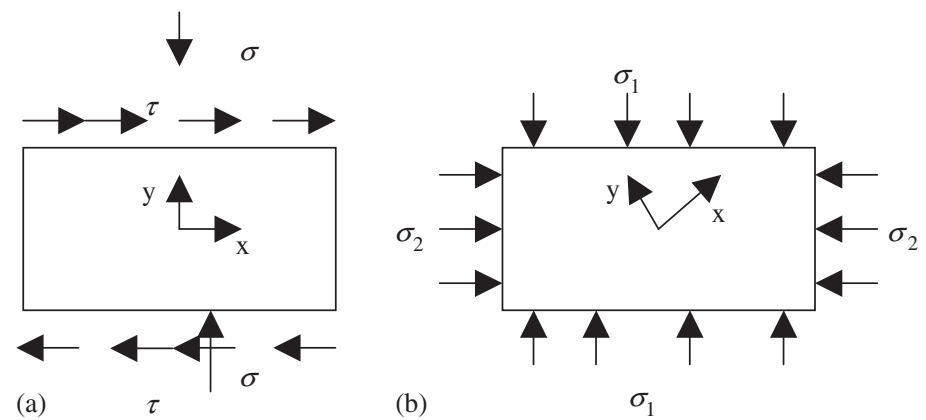

Figure 1. (a) The sketch of simple shear; and (b) the sketch of plane strain state.

\section{THE CONSTITUTIVE RELATIONS}

The skeleton of soils is taken as visco-plastic, so the constitutive relations may be expressed as follows under shear loading [30]:

$$
\left\{\begin{array}{l}
\sigma_{x}^{\prime}=f_{1}(\gamma, \dot{\gamma}, u) \\
\sigma_{y}^{\prime}=f_{2}(\gamma, \dot{\gamma}, u) \\
\tau=f_{3}(\gamma, \dot{\gamma}, u)
\end{array}\right.
$$

in which $\sigma_{x}^{\prime}, \sigma_{y}^{\prime}$ are effective stresses in $x$ and $y$ directions, respectively, $\tau$ is the shear stress, $\gamma$ is the shear strain, $\dot{\gamma}$ is the shear strain rate, $u$ is the pore pressure and is equal to $\sigma-\sigma_{e y}, \sigma$ is the total stress, it is a constant in simple shear, which means that, $u$ is equivalence to $\sigma_{y}^{\prime}$ in this case.

\section{ONE-DIMENSIONAL PERTURBATION ANALYSIS}

$\mathrm{Lu}$ [30] has used linear perturbation analysis to examine the growth of infinitesimal inhomogeneities in homogeneous simple shear. These analyses include strain hardening, pore pressure softening, etc. Following Lu's analysis, the controlling equations are as follows:

$$
\begin{aligned}
& \frac{\partial^{2} \tau}{\partial y^{2}}=(1-n) \rho_{s} \frac{\partial^{2} \gamma}{\partial t^{2}}+K n^{2} \frac{\partial \gamma}{\partial t} \\
& C \dot{\gamma}=-\frac{1}{K n} \frac{\partial^{2} u}{\partial y^{2}}+\frac{1}{E_{r}} \frac{\partial u}{\partial t}
\end{aligned}
$$

in which $\tau$ is the shear stress, $n$ is porosity, $\rho_{s}$ is the density of grains, $\gamma$ is the shear strain, $K$ is the obstruction coefficient whose dimension is $\left[L^{2}\right]$ and $K=\mu / k_{1}$, where $k_{1}$ is the physical permeability and $\mu$ is the viscosity, $C$ is the coefficient denoting dilatancy, $u$ is the pore pressure, $E_{r}$ is the compressible coefficient of the grains, $t$ is the time and $y$ is the co-ordinate. 


\section{INSTABILITY CONDITIONS}

In the above equations, the $y$-axis is taken to be normal to the shear band length. Considering disturbances of the form

$$
\left\{\begin{array}{l}
\gamma=\gamma^{\circ}+\gamma^{\prime} \exp (\alpha t+\mathrm{i} k y) \\
p=p^{\circ}+p^{\prime} \exp (\alpha t+\mathrm{i} k y) \\
\tau=\tau^{\circ}+\tau^{\prime} \exp (\alpha t+\mathrm{i} k y)
\end{array}\right.
$$

in which $\alpha$ and $k$ are, respectively, the frequency and the wave number. $\gamma^{\circ}, p^{\circ}, \tau^{\circ}$ is a solution to the system of Equation (2), and $\left|\gamma^{\prime}\right| \ll\left|\gamma^{\circ}\right|,\left|p^{\prime}\right| \ll\left|p^{\circ}\right|,\left|\tau^{\prime}\right| \ll\left|\tau^{\circ}\right|$.

By the third Equation of (1), we can obtain

$$
\begin{aligned}
\tau^{*} & =\mathrm{d} \tau_{0}=\left(\frac{\partial f}{\partial \varepsilon}\right)_{0} \mathrm{~d} \varepsilon+\left(\frac{\partial f}{\partial \dot{\varepsilon}}\right)_{0} \mathrm{~d} \dot{\varepsilon}-\left(-\frac{\partial f}{\partial p}\right)_{0} \mathrm{~d} p \\
& =R_{0} \gamma^{*}+H_{0} \alpha \gamma^{*}-Q_{0} p^{*}
\end{aligned}
$$

in which $R_{0}$ is strain hardening coefficient, $Q_{0}$ is strain rate hardening coefficient, $H_{0}$ is pore pressure softening coefficient.

Substituting Equation (3) in Equation (2) and considering Equation (4), a homogeneous system of equations about $\gamma^{\prime}, p^{\prime}$ may be obtained. The determinant of coefficients must be equal to zero if the equation have a non-trivial solution, which leads to the following spectral equation [30].

$$
(1-n) \rho_{s} \alpha^{3}+\left(\frac{(1-n) \rho_{s} E_{r}}{K n} \beta^{2}+H_{0} \beta^{2}+K n^{2}\right) \alpha^{2}+A_{1} \alpha+\frac{E_{r} R_{0}}{K n} \beta^{4}=0
$$

in which $A_{1}=\left(E_{r} H_{0} / K n\right) \beta^{4}+E_{r} n \beta^{2}+R_{0} \beta^{2}-E_{r} C Q_{0} \beta^{2}$.

Now the following variables are adopted:

$$
\alpha=\frac{K}{\rho_{s}} \bar{\alpha}, \quad \beta^{2}=\frac{K^{2}}{\rho_{s} R_{0}} \bar{\beta}_{2}, \quad A=\frac{R_{0}}{E_{r}}, \quad B=\frac{H_{0} K}{\rho_{s} E_{r}}, \quad C_{1}=C Q_{0}
$$

Substituting the above variables in Equation (5), the dimensionless spectral equation may be obtained:

$$
n(1-n) A \bar{\alpha}^{3}+\left\lfloor(1-n+n B) \bar{\beta}^{2}+A n^{3}\right\rfloor \bar{\alpha}^{2}+\left\lfloor\left(n^{2}+n A-n C\right) \bar{\beta}^{2}+A B \bar{\beta}^{4}\right\rfloor \bar{\alpha}+\bar{\beta}^{4}=0
$$

It is easy to find that in the case of long wavelength $(\bar{\beta} \rightarrow 0)$ and short wavelength $(\bar{\beta} \rightarrow \infty)$, Equation (7) has negative roots, which means that, the deformation is always stable. But the negative term $n^{2}+n A-n C$ which may lead to instability must occur at spectral wave numbers. Therefore, it is interested to seek the wave number $\bar{\beta}_{m}$ for which the corresponding $\bar{\alpha}_{m}>0$ is a maximum. Thus $\bar{\beta}_{m}$ and $\bar{\alpha}_{m}$ must satisfy the next equation

$$
\mathrm{d} \bar{\alpha} / \mathrm{d} \bar{\beta}^{2}=0
$$

which leads to

$$
\bar{\beta}_{m}^{2}=-\frac{(1+n+n B) \bar{\alpha}_{m}^{2}+\left[n^{2}+n(A-C)\right] \bar{\alpha}_{m}}{2\left(A B \bar{\alpha}_{m}+1\right)}
$$


Substituting the above equation in Equation (7), the equation for determining $\bar{\alpha}_{m}$ may be obtained as follows:

$$
4 A n\left[(1-n) \bar{\alpha}_{m}+n^{2}\right]\left(A B \bar{\alpha}_{m}+1\right)=\left\{(1+n+n B) \bar{\alpha}_{m}+\left[n^{2}+n(A-C)\right]\right\}^{2}
$$

It is obvious that if the following condition is satisfied, the above equation have solutions in the range $\bar{\alpha}_{m}>0$ :

$$
\frac{A-2 \sqrt{A n}+n}{C}<\frac{A}{C}<1
$$

which is

$$
\frac{R_{0}}{E_{r} C Q_{0}}<1
$$

It is shown that the instability occurs when the strain hardening effect overcomes the pore pressure softening effect.

\section{THE THICKNESS OF SHEAR BAND UNDER SIMPLE SHEAR}

As shear bands eventually take on a characteristic thickness with increasing time, from the controlling equations as $t \rightarrow \infty$ an estimation of the thickness $\delta$ may be obtained as follows [31,32]:

$$
C \dot{\gamma} \approx\left|\frac{1}{K n} \frac{\partial^{2} u}{\partial y^{2}}\right|
$$

An estimate of the term $1 / K n \partial^{2} u / \partial y^{2}$ is

$$
\left|\frac{1}{K n} \frac{\partial^{2} u}{\partial y^{2}}\right| \approx \frac{1}{K n}\left(\frac{u_{*}-u_{m}}{\delta^{2}}\right)
$$

in which $u_{*}, u_{m}$ are the pore pressure within the shear band and surrounding the shear band respectively. Now because $u_{*} \gg u_{m}$, it is possible to write the following simplified equation for the shear band half thickness:

$$
\delta=\left(\frac{u_{*}}{C_{1} \dot{\gamma}_{*} K n}\right)^{0.5}
$$

At high pore pressure or low effective stress, the material viscosity may be important. Then, assuming $\dot{\gamma}_{*}=\tau_{*} / \eta$ and therefore the equation above becomes

$$
\delta=\left(\frac{\eta u_{*}}{C_{1} K n \tau^{*}}\right)^{0.5}
$$

From this equation, it may be concluded that the shear band thickness in saturated soils should be a material whose characteristics are dependent on the physical and mechanical properties of the material. 


\section{MULTIDIMENSIONAL SHEAR BAND THICKNESS}

Except in special cases, such as simple shear, shear bands in saturated soils are not formed in one dimension. An obvious example of shear band in more complex stress states is plane strain compression which can be regarded as a pure shear with a superimposed hydrostatic pressure. There is direct experimental evidence that a superimposed hydrostatic pressure does not effect the formation of shear band, but only the late stages which involve the formation of microcracks within the bands (e.g. Reference [7]). Thus, it may well be that hydrostatic pressure does not influence the shear band thicknesss formed.

For multidimensional bands, the controlling equations becomes the same as in the literature [30].

$$
C\left(\dot{\gamma}_{x y}+\dot{\gamma}_{x z}+\dot{\gamma}_{x z}\right)=-\frac{1}{K n}\left(\frac{\partial^{2} u}{\partial x^{2}}+\frac{\partial^{2} u}{\partial y^{2}}+\frac{\partial^{2} u}{\partial z^{2}}\right)+\frac{1}{E_{r}} \frac{\partial u}{\partial t}
$$

in which $\dot{\gamma}_{x y}, \dot{\gamma}_{x z}$, and $\dot{\gamma}_{y z}$ are the shear strain rates in $x-y, x-z$, and $y-z$ planes, respectively.

It has been found that the late stage of shear band formation is pore pressure diffusion dominated unlike the inertia dominated initial stages [33]. Once the band is formed, the overall deformation will reduce to a plane strain stress state. These assumptions lead to the following two-dimensional momentum equations:

$$
C\left(\dot{\gamma}_{x y}+\dot{\gamma}_{x z}\right)=-\frac{1}{K n}\left(\frac{\partial^{2} u}{\partial x^{2}}+\frac{\partial^{2} u}{\partial y^{2}}\right)+\frac{1}{E_{r}} \frac{\partial u}{\partial t}
$$

Now, the effect of a variation in pore pressure in relation to one shear band is investigated. If one shear band is considered, and thickness $\ll l, l$ is the characteristic length of the sample, the controlling equation becomes

$$
C\left(\dot{\gamma}_{x y}+\dot{\gamma}_{x z}\right)=-\frac{\Delta p}{K n \delta^{2}} \frac{\partial^{2} \bar{u}}{\partial \bar{y}^{2}}+\frac{\Delta \bar{u}}{E_{r} t_{k}} \frac{\partial \bar{u}}{\partial t}
$$

in which

$$
\bar{y}=\frac{y}{\delta} \approx 0(1), \quad \bar{u}=\frac{u}{\Delta u} \approx 0(1), \quad \bar{t}=\frac{t}{t_{k}} \approx 0(1)
$$

in which $t_{k}$ is the characteristic time, $\Delta u$ is the pore pressure increase. Over the time $t_{k}$ the pore pressure variation $\Delta u$ occurs, hence

$$
t_{k} \approx \frac{\delta^{2}}{\left(E_{r} / K\right)}
$$

and therefore

$$
\frac{\partial \bar{u}}{\partial \bar{t}} \approx \frac{\partial^{2} \bar{u}}{\partial \bar{y}^{2}} \approx 0(1)
$$

Now the controlling equation reduces to the following case:

$$
\frac{1}{t_{k}} \frac{\partial \bar{u}}{\partial \bar{t}} \approx \frac{C\left(\dot{\gamma}_{x y}+\dot{\gamma}_{x z}\right)}{\Delta u}+\frac{E_{r}}{K n} \frac{1}{\delta^{2}} \frac{\partial^{2} \bar{u}}{\partial \bar{y}^{2}}
$$


This assumption requires that the time at which the normal strains parallel and perpendicular to the band are developed must be greater than the diffusion time across the band thickness. This is obviously satisfied for shearing in saturated soils under undrained conditions. Thus, the conclusion is that the thickness of shear band formed under a combined stress state is mainly dominated by the one-dimensional equation for simple shear after considering the dilatancy rate resulting from the combined stress state. Alternatively, the thickness of shear band can be considered to be primarily dependent upon the strain rate and pore pressure properties and to a lesser extent on the constitutive relation. The generalized formulae may be written as

$$
\delta \approx\left(\frac{E_{r} \Delta u_{*}}{C\left(\dot{\gamma}_{x y}+\dot{\gamma}_{x z}\right) K n}\right)^{0.5}
$$

\section{THE COMPARISON WITH THE EXPERIMENTAL DATA}

The apparent independence of shear band thickness is supported by some experiments. The data adopted are given a simple description here:

Alshibli and Sture [6] used digital imaging analysis to study localized deformations in granular materials tested under plane strain condition. The shear band thickness are from 3.19 to $3.29 \mathrm{~mm}$. The material used in their experiments was a fine-grained sand (F-75 Silica sand). The mean particle size is equal to $0.22 \mathrm{~mm}$, The specific gravity of the particles is 2.65 . The porosity is about 0.5 . The confining pressures are 15 and $100 \mathrm{kPa}$. They processed their experiments using plane strain compression. The strain rate is assumed to be $4 \times 10^{-4} \mathrm{~s}$. $E_{r}=$ $10^{5} \mathrm{~Pa}$, the obstruction coefficient is equal to $K=10^{11} \mathrm{~kg} / \mathrm{m}^{3} \mathrm{~s}$, the porosity is about 0.6 . The pore pressure increase is about $10^{5} \mathrm{~Pa}$.

Oda and Kazama [7] carried plane strain experiments. It is shown that the shear band thickness is about 7-8 times the mean particle size. The material they adopted is Toyoura sand. Toyoura sand is a uniform dune sand with a mean particle size $0.206 \mathrm{~mm}$. The shear strain rate is about $0.0012 \%$ while the minor stress $\sigma_{3}$ was kept as a constant value. $E_{r}=10^{4} \mathrm{~Pa}$, the obstruction coefficient is equal to $K=10^{12} \mathrm{~kg} / \mathrm{m}^{3} \mathrm{~s}$. The porosity is about 0.6 , The pore pressure increase is about $10^{5} \mathrm{~Pa}$.

In the Nemat-Nasser's [34] triaxial torsion apparatus experiments, two types of sand, Silica No. 60 and Monterey No. 0, are used to examine the particle-size effect on shear localization. The mean particle diameters of Monterey No. 0 and Silica No. 60 are 0.48 and $0.22 \mathrm{~mm}$, respectively. The shear band thickness is 7.0 and $2.5 \mathrm{~mm}$, respectively. The samples are consolidated under 294 and $196 \mathrm{kN} / \mathrm{m}^{2}$ effective pressures. The shear strain rate is 0.0017 and $0.83 \%$. The porosity is about 0.35 and 0.46 . It is shown that the band thickness is about $10-15$ times as large as the mean particle diameter. The pore pressure increase is about $10^{5} \mathrm{~Pa}$. The parameter $E_{r}$ is equal to $10^{6}$ and $10^{7} \mathrm{~Pa}$ for Monterey No. 0 and Silica No. 60, respectively, $K$ is equal to about $10^{11} \mathrm{~kg} / \mathrm{m}^{3} \mathrm{~s}$.

The comparison between our results and the above experimental data are given in Table I. The parameter $C$ is estimated by the fact that each term in the second equation of (2) should be in the same order. $C$ is adopted to equal $10^{6}-10^{7} \mathrm{~Pa}$ in the computing. It is shown that they are close to each other. 
Table I. Comparison of calculated and experimental shear band thickness.

\begin{tabular}{lcccc}
\hline Material & Loading conditions & Band thickness $/$ D $_{50}$ & Ref. & Computed data \\
\hline Sand & Plane strain & $7-8$ & {$[6]$} & 8 \\
Sand & Plane strain & $14-15$ & {$[7]$} & 13 \\
Soil & Triaxial torsion & $10-15$ & {$[31]$} & $10-12$ \\
\hline
\end{tabular}

\section{CONCLUSION}

A formula for the thickness of a shear band formed in saturated soils under simple shear and combined stress state has been proposed.

In the controlling equations, the dilatancy, the pore pressure dissipation, the inertial and the obstruction between the grains and water are considered. The stresses are considered as the function of strain, strain rate and pore pressure.

Perturbation method is used to seek for the instability conditions. It is shown that the instability occurs when the strain hardening effect overcomes the pore pressure softening effect. A finite thick shear band may develop after instability. Based on the fact that the shear band formation is in a late stage and so the initial may be neglected, the approximated formulas of bandwidth are obtained. It is shown that the shear band thickness is dependent on the pore pressure properties of the material and the dilatancy rate, but is independent of the details of the combined stress state. This is in accordance with some separate experimental observations.

\section{ACKNOWLEDGEMENTS}

This programme is supported by the National Natural Science Foundation-Key Youth Foundation (No. 40025103) and National Natural Science Foundation (No. 10202024).

\section{REFERENCES}

1. Iai S, Bardet JP. Plane strain instability of saturated elasto-plastic soils. Geotechnique 2001; 51(5):389-398.

2. Lade PV. Experimental observations of stability, instability and shear planes in granular materials. Ingenieur Archiv 1989; 59:114-123.

3. Matthias L, Christian M. A note on formulas for localised failure of frictional materials in compression and biaxial loading modes. International Journal for Numerical and Analytical Methods in Geomechanics 2001; 25:955-971.

4. Finno RJ, Harris WW, Mooney MA, Viggiani G. Strain localization and undrained steady state of sand. Journal of Geotechnological Engineering 1996; 122(6):462-473.

5. Alshibli KA, Sture S. Shear band formation in plane strain experiments of sand. Journal of Geotechnological and Geoenvironmental Engineering (ASCE) 2000; 126(6):495-503.

6. Alshibli KA, Sture S. Sand shear band thickness measurements by digital imaging techniques. Journal of Computing in Civil Engineering (ASCE) 1999; 13(2):103-109.

7. Oda M, Kazama H. Microstructure of shear bands and its relation to the mechanism of dilatancy and failure of dense granular soils. Geotechnique 1998; 48(4):465-481.

8. Nemat-Nasser S, Zhang JH. Constitutive relations for cohesionless frictional granular materials. International Journal of Plasticity 2002; 18(4):531-547.

9. Lade PV, Qiong Wang. Effects of shear banding on three-dimensional failure conditions for soils. In Numerical Models in Geomechanics - NUMOG VII, Pande, Pietruszczak S, Schweiger HF (eds). Balkema: Rotterdam, 1999.

10. Mooney MA, Finno RJ, Viggiani MG. A unique critical state for sand. Journal of Geotechnical and Geoenvironmental Engineering (ASCE) 1998; 124(11):1100-1108. 
11. Borja IB, Lai TY. Propagation of localization instability under active and passive loading. Journal of Geotechnical and Geoenvironmental Engineering 2002; 128(1):64-75.

12. Rice JR. On the stability of dilatant hardening of saturated rock masses. Journal of Geophysical Research 1975; 80:1531-1536.

13. Muhlhaus HB, Boland J. The influence of microstructure-induced gradients on the localization of deformation in viscoplastic materials. Acta Mechanica 1991; 89(1-4):217-231.

14. Rudnicki JW, Rice JR. Conditions for the localization of deformation in pressure-sensitive dilatant material. Journal of the Mechanics and Physics of Solids 1975; 23:371-394.

15. Li XS. Modelling of dilative shear failure. Journal of Geotechnical and Geoenvironmental Engineering (ASCE) 1997; 123(7):609-616.

16. Pietruszczak S, Niu X. On the description of localized deformation. International Journal for Numerical and Analytical Methods in Geomechanics 1993; 17:791-805.

17. Pietruszczak S. Undrained response of granular soil involving localized deformation. Journal of Engineering Mechanics (ASCE) 1995; 121(12):1292-1297.

18. Loret B, Prevost JH. Dynamic strain localization in fluid-saturated porous media. Journal of Engineering Mechanics (ASCE) 1991; 117(4):907-923.

19. Vardoulakis I. Stability and bifurcation of undrained, plane rectilinear deformations on water-saturated granular soils. International Journal for Numerical and Analytical Methods in Geomechanics 1985; 9:399-414.

20. Vardoulakis I. Shear-banding and liquefaction in granular materials on the basis of a Cosserat continuum theory. Ingenieur Archiv 1989; 59:106-113.

21. Atkinson JH. Non-linear soil stiffness in routine design. Geotechnique 2000; 50(5):487-508.

22. Muhlhaus HB, Vardoulakis I. The thickness of shear bands in granular materials. Geotechnique 1987: 37(3): 271-283.

23. de Borst R. Embedded discontinuity approaches for shear band analysis. In Numerical Models in Geomechanics-NUMOG VII, Pande, Pietruszczak S, Schweiger HF (eds). Balkema: Rotterdam, 1999; 103-108.

24. Lu XB, Peng Cui. The influence of strain gradient on the shear band in a saturated soil. Iranian Journal of Science and Technology 2003; 27(A1):57-62.

25. Lu Xiaobing, Zhang Jiahua, Yang Zhengsheng. On the evolution of shear bands in saturated soils. International Journal of Non-Linear Mechanics 2000; 35:21-26.

26. Lesniewska D, Mroz Z. Limit equilibrium approach to study the evolution of shear band systems in soils. Geotechnique 2000; 50(5):521-536.

27. Tejchman J. Patterns of shear zones in granular bodies within a polar hypoplastic continuum. Acta Mechanica 2002; 155:71-79.

28. Wei Wu. Non-linear analysis of shear band formation in sand. International Journal for Numerical and Analytical Methods in Geomechanics 2000; 24:245-263.

29. Vardoulakis I, Aifantis EC. Gradient dependent dilatancy and its implications in shear banding and liquefaction. Ingenieur Archiv 1989; 59:197-208.

30. Lu XB. On the shear instability of saturated soil. International Journal of Engineering Science 2001; 39:963-972.

31. Dodd B, Bai Y. Width of adiabatic shear bands. Materials Science and Technology 1985; 1:38-40.

32. Dodd B, Bai Y. Width of adiabatic shear bands formed under combined stresses. Materials Science and Technology 1989; 5:557-559.

33. Lu XB, Wang SY. On the evolution of simple shear in saturated soils. International Journal for Numerical and Analytical Methods in Geomechanics 2004; 28:269-278.

34. Nemat-Nasser S, Okada N. Radiographic and microscopic observation of shear bands in granular materials. Geotechnique 2001; 51(9):753-765.

35. Atkinson JH, Richardson D. The effect of local drainage in shear zones on the undrained strength of overconsolidated clay. Geotechnique 1987; 37(3):393-403. 